\title{
Numerical Methods for Solving the Dynamic Behavior of Real Systems
}

\author{
V. Nikolić, D. Djekić, A. Radaković, Dž. Pučić
}

\begin{abstract}
In this paper are shown dynamic models which represent the real systems. As an example, one gear couple has been presented. In this case, the simplified model two discrete masses with elastic and damped connections. Using the of mechanics and taking into account initial and basic principles conditions, the system of differential equations is established which describes physicality of motion. Such system of equations represents mathematical model at dynamical behavior of gear transmission. Established system of differential equations has been solved in analytical way with the aim of obtaining data for numerical analysis. The proper estimate of dynamical behavior of gear transmission and other systems can be on the basis of own frequency and of own form of oscillating. In the paper is presented the solution of the main form of oscillating and stress condition using finite elements method.
\end{abstract}

Keywords: Stress and strain state analysis, tensor invariants, Finite Element Method, Geared transmissions.

\section{Introduction}

In solving dynamic problems, a real engineering system is replaced by a simplified dynamic model. This model consists of continually or discretely positioned masses with elastic and damped connections among individual members. A system of equations describing physicality of the process of motion is set by applying the basic principles of mechanics and taking into consideration the initial and boundary conditions. That system of equations represents the mathematical model of the machine or system in dynamic behavior.

The equations of motion of dynamic systems which are analog to the equations of equilibrium in static analysis, can be derived on the basic of D'Alambert principle, the principle of virtual displacements or Hamilton principle [1,2,3].

Manuscript received April 7, 2013; accepted September 11, 2013.

V. Nikolić, D. Djekić, A. Radaković, Dž. Pučić are with the State University of Novi Pazar, Novi Pazar, Serbia. 
In order to minimize the error in modeling dynamic systems, it is necessary to represent the system with a greater number of masses, i.e., degrees of freedom. On the other hand, increased number of degrees of freedom makes the mathematical model more complex and solving of the given system of equations more difficult.

Taking the exact nature of the discussed problem into consideration, during this research the Finite Elements Method (FEM) has been chosen as one of the numerical methods, $[1,2,3,9,10]$.

\section{Equations that describe the dynamic behavior of the system}

To formulate the equations of motion in the finite method, we start from Hamilton variation principle and functional Lagrange, 2,3,4 .

Taking into account the equations for kinetic and potential energy [1,2,3], displacements of velocity, acceleration and deformation in the finite element expressed through interpolation functions, as well as the expression for damping forces, the matrix equation of motion of the finite element is obtained in the following form

$$
\mathbf{m} \ddot{q_{e}}+c \dot{q}_{e}+k q_{e}=Q_{e}
$$

In this equation are

$$
\begin{array}{r}
\mathbf{m}=\int_{\mathbf{v}} \mathbf{N}^{\mathbf{T}} \rho \mathbf{N} d V \text { matrix of mass of the element, } \\
\mathbf{c}=\int_{\mathbf{v}} \mathbf{N}^{\mathbf{T}} \mathbf{c} \mathbf{N} d V \text { matrix of damping of the element, } \\
\mathbf{k}=\int_{\mathbf{v}} \mathbf{B}^{\mathbf{T}} \mathbf{D} \mathbf{B} d V \text { stiffness matrix of the element, } \\
\mathbf{Q}_{\mathbf{e}}=\int_{\mathbf{v}} \mathbf{N}^{\mathbf{T}} \mathbf{F} d V+\int_{\mathbf{S}} \mathbf{N}^{\mathbf{T}} \mathbf{F}_{\mathbf{n}} d S \text { vector of generalized forces }
\end{array}
$$

The equation of motion for the finite element system, that is, the structure, is formed on the basic of the matrix equations of motion for individual finite elements

$$
\mathbf{M} \ddot{\mathbf{q}}+\mathbf{C} \dot{\mathbf{q}}+\mathbf{K q}=\mathbf{Q}
$$

where $\mathbf{M}, \mathbf{C}$ i $\mathbf{K}$ matrices of mass, damping and stiffness of the structure, and $\mathbf{Q}$ is vector of generalized forces in the nodes of the finite element system.

Determination of own vibrations of structural elements is a separate problem often referred to as the problem of own values or eigne problem. The result of studying free oscillations of the system are own frequencies and own vectors. The simplest case is the system without damping and action of external forces so it is described by a differential equation

$$
\mathbf{M} \ddot{\mathbf{q}}+\mathbf{K q}=0 .
$$


If the vector of generalized nodal displacements is expressed in the form of

$$
\mathbf{q}=\overline{\mathbf{q}} e^{i w t}, e^{i w t}=\cos w t+i \sin w t
$$

then the matrix equation has the form of

$$
\left(\mathbf{K}-w^{2} \mathbf{M}\right) \overline{\mathbf{q}}=0
$$

and it is a system of algebraic equations per unknown amplitudes $\overline{\mathbf{q}}$. In order for this system of equations to have solutions (apart from the trivial $\mathbf{q}=0$ ), it is necessary to have the determinant of the system equal to zero, that is

$$
\left|\mathbf{K}-w^{2} \mathbf{M}\right|=0
$$

This equation is known as the characteristic equation of the system. Solutions of this equation (roots) $w_{i}^{2}$, of which generally there are $n$, are represented by the squares of own frequencies of the system or own values of the system which we usually arrange by increasing order $w_{1}^{2}<w_{2}^{2}<w_{3}^{2}<\ldots<w_{n}^{2}$. For each own value there is an own vector $\overline{\mathbf{q}}$ which represents the own form or node of vibrations.

The vectors of natural or own forms possess the characteristic of modal orthogonality which can be expressed by the term

$$
\overline{\mathbf{q}}_{\mathbf{i}}^{\mathbf{T}} \mathbf{M} \overline{\mathbf{q}}_{\mathbf{j}}=\delta_{i j}=\left\{\begin{array}{ll}
1, & i=j \\
0, & i \neq j
\end{array}\right\} \quad \mathrm{i} \quad \overline{\mathbf{q}}_{\mathbf{i}}^{\mathbf{T}} \mathbf{K} \overline{\mathbf{q}}_{\mathbf{j}}=w_{i}{ }^{2} \delta_{i j}
$$

so, if the vectors $\overline{\mathbf{q}}_{\mathbf{i}}$ are arranged in such a way that they form columns of the matrix, then the matrix obtained represents the modal matrix which is used to transform generalized coordinates into main coordinates.

As a real practical examples on which will be applied to developed the theory and FEM were selected gear transmissions.

\section{Dynamic models of gear transmission}

Dynamic behavior of gear transmission is primarily the result of changes of external load as well as of occurrence of internal dynamic forces. The change of external load originates from the change of force of the power engine and also from the very fact that in engagement single and double mesh are alternated $[2,9,10]$. Additional internal dynamic forces depend on rigidity of teeth, displacement of profile, manufacturing errors (mesh, pitch, shape and the others) peripheral velocity, mass of rotary parts rigidity of twisting and bending of the shaft, etc. Also, internal dynamic forces occur due to elastic deformations of the teeth. The occurrence of internal dynamic forces has been studied for years. Further in the text a model of oscillation of a gear set will be described. 
On the basic of Lagrange equations of other types differential equations of second order are written and solving of this system defines the dynamic process of discrete points.

Coupled gears, or single-step gear transmission can be represented by an oscillatory model, Fig. 1a, $[1 \mathrm{~b}, 2 \mathrm{a}, 5,6,7]$, where $F_{n}(t)$ denotes the initiating force, while $m_{r 1}$ and $m_{r 2}$ are reduced masses of the gears. A simplified dynamic model of the gear set is shown on Fig. $1 b$.

Reduced masses of individual gears are

$$
m_{r 1}=\frac{J_{1}}{r_{b 1}^{2}}, m_{r 2}=\frac{J_{2}}{r_{b 2}^{2}}
$$

where $J_{1}$ and $J_{2}$ moments of inertia of rotary masses and $r_{b 1}$ and $r_{b 2}$ are radii of base circles at gears 1 and 2 .

The initiating force is of the following form: $F_{n}(t)=F_{0}+F_{1} \sin \Omega_{1} t_{1}$.

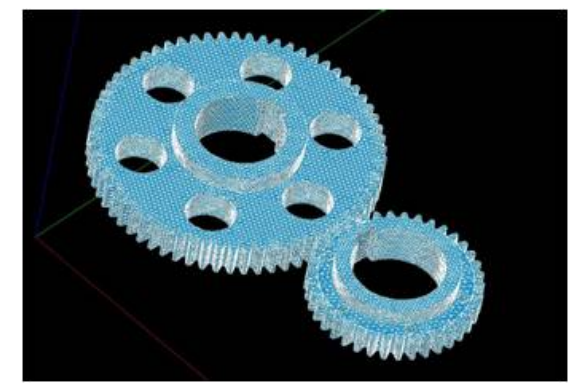

(a)

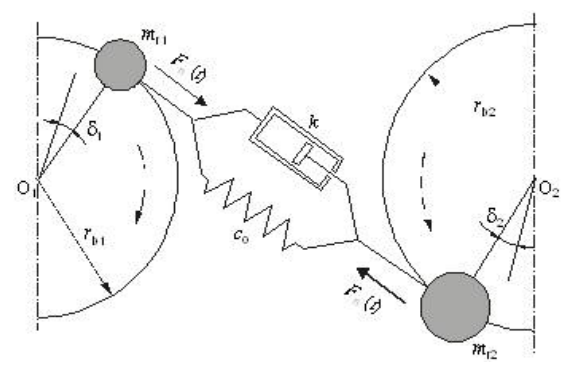

(b)

Figure 1: (a) Gear set FME model; (b) A simplified dynamic model of the gear set

It means that the teeth mesh is replaced by the action of a variable force at teeth flanks $F_{n}(t)$ which changes in proportion to the deformation of teeth in mesh. It is assumed here in that rigidity of the teeth in mesh is equal to medium rigidity $c_{0}$.

By applying the above described procedure, we can demonstrate that this model is described by a system of differential equation, $[2,6,7,8,9,10]$

$$
\begin{aligned}
& m_{r 1} \ddot{x}_{1}+k\left(\dot{x}_{1}-\dot{x}_{2}\right)+c_{0}\left(x_{1}-x_{2}\right)=F_{n}(T), \\
& m_{r 2} \ddot{x}_{2}-k\left(\dot{x}_{1}-\dot{x}_{2}\right)-c_{0}\left(x_{1}-x_{2}\right)=-F_{n}(T) .
\end{aligned}
$$

In case the gear supports $O_{1}$ and $O_{2}$ are absolutely rigid, the total displacement in the direction of the contact line will be $x=x_{1}-x_{2}$ and $k$ is gear mesh dumping functions depending on $t$. 
The corresponding matrices for the above system, that is the matrix of damping coefficient $\mathbf{C}$, matrix of elasticity $\mathbf{K}$ and matrix of inertia $\mathbf{M}$, have the following forms

$$
\mathbf{C}=\left[\begin{array}{cc}
k & -k \\
-k & k
\end{array}\right] ; \quad \mathbf{K}=\left[\begin{array}{cc}
c_{0} & -c_{0} \\
-c_{0} & c_{0}
\end{array}\right] ; \quad \mathbf{M}=\left[\begin{array}{cc}
m_{r 1} & \\
& m_{r 2}
\end{array}\right] .
$$

Let us assume the homogenous part of the solution of this system in the form

$$
\left\{\begin{array}{l}
x_{1} \\
x_{2}
\end{array}\right\}=\left\{\begin{array}{l}
A_{1} \\
A_{2}
\end{array}\right\} e^{\lambda t}
$$

which, by substituting in (17) gives the system of algebraic equations which has the characteristic polynom

$$
f(x)=\left|\begin{array}{cc}
m_{r 1} \lambda^{2}+k \lambda+c_{0} & -c_{0}-k \lambda \\
-k \lambda-c_{0} & m_{r 2} \lambda^{2}+k \lambda+c_{0}
\end{array}\right|
$$

On Fig. 2a is dynamic model of the transmission with four degrees of freedom is used and simplified dynamic model of the gear set is shown on Fig. $2 b$.

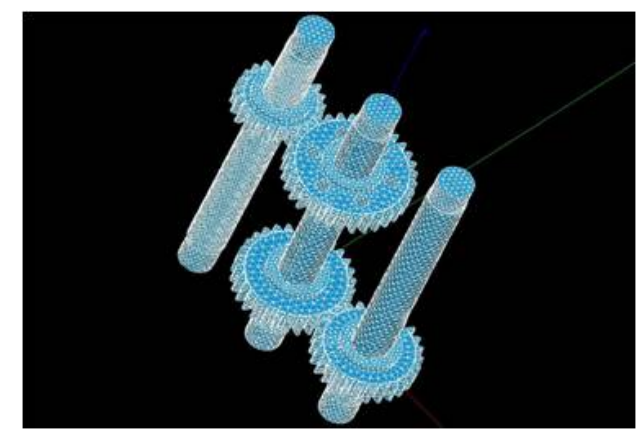

(a)

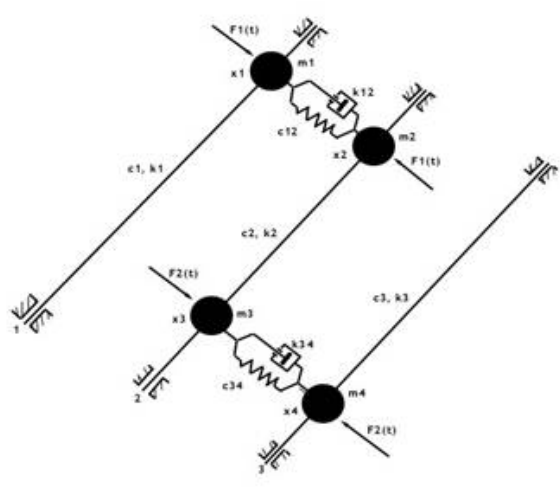

(b)

Figure 2: (a) Gear transmission model with four degrees of freedom. (b)A simplified dynamic model of the geared transmission.

The differential equation, $[2,8,9,10]$, of the model on Fig. 2a are:

$$
\begin{aligned}
m_{1} \ddot{x_{1}}+\left(c_{1}+c_{12}\right) x_{1}-c_{12} x_{2}+\left(k_{1}+k_{12}\right) \dot{x_{1}}-k_{12} \dot{x_{2}} & =F_{1}(t), \\
m_{2} \ddot{x_{2}}+\left(c_{2}+c_{12}\right) x_{2}-c_{12} x_{1}-c_{2} x_{3}+\left(k_{2}+k_{12}\right) \dot{x_{2}}-k_{12} \dot{x_{1}}-k_{2} \dot{x_{3}} & =-F_{1}(t), \\
m_{3} \ddot{x_{3}}+\left(c_{2}+c_{34}\right) x_{3}-c_{34} x_{4}-c_{2} x_{2}+\left(k_{2}+k_{34}\right) \dot{x_{3}}-k_{34} \dot{x_{4}}-k_{2} \dot{x_{2}} & =F_{2}(t), \\
m_{4} \ddot{x_{4}}+\left(c_{3}+c_{34}\right) x_{4}-c_{34} x_{3}+\left(k_{3}+k_{34}\right) \dot{x_{4}}-k_{34} \dot{x_{3}} & =-F_{2}(t)
\end{aligned}
$$

where: 
- $m_{i}(i=1,4)$ are equivalent masses of transmission elements,

- $c_{i}$ are shaft stiffness $(i=1,3)$,

- $k_{i}$ are shaft dumping $(i=1,3)$

- $c_{12}, c_{34}$ are gear mesh stiffness functions depending on $t$,

- $k_{12}, k_{34}$ are gear mesh dumping functions depending on $t$.

Shaft stiffness/dumping outside of the gears on the shaft 2 are not considered.

\section{Presentation of results}

As an example for modal analysis is taken a single-step gear set (Fig. 1a) with the following data, Table 1:

Table 1: Data for gear set

\begin{tabular}{|c|c|c|}
\hline Parameter & Gear 1 & Gear 1 \\
\hline Number of teeth & 38 & 65 \\
\hline Modul & $0,003 \mathrm{~m}$ & $0,003 \mathrm{~m}$ \\
\hline Standard angle & $20^{\circ}$ & $20^{\circ}$ \\
\hline Material & Č44320 & Č4320 \\
\hline
\end{tabular}

Boundary conditions have been defined per displacement (all displacements at gear and shaft are equal to zero), Fig. 3. The load is specified in contact points of the gears (drive power is $P=7,5 \mathrm{~kW}$ and revolution number $n=800 \mathrm{~min}^{-1}$ ).

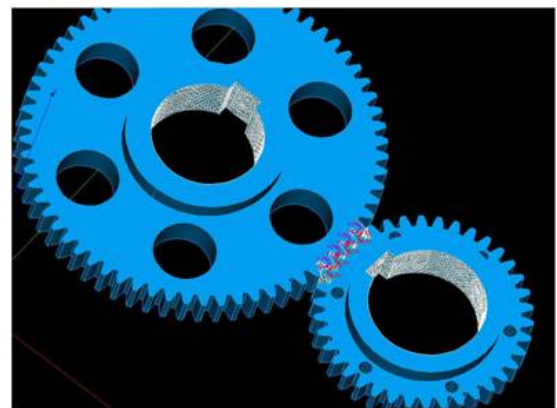

Figure 3: Displacement and force boundary conditions

The first two main shapes of oscillation of the system of two gears are shown in Fig. 4 (a), 4 (b), etc. 


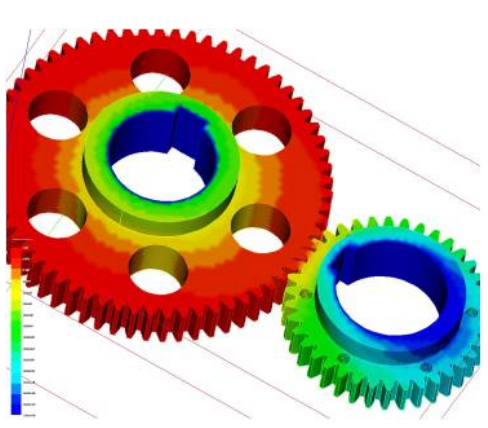

(a)

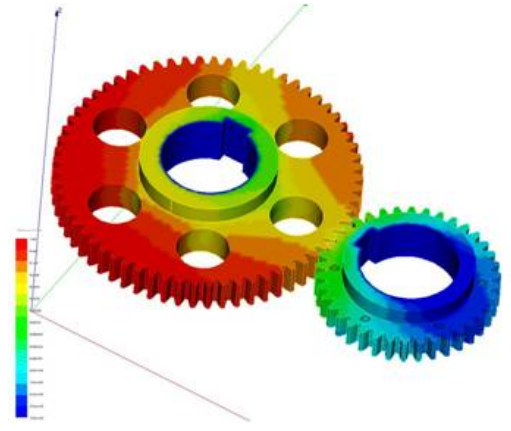

(b)

Figure 4: (a) First mode shape (f=1631.48 Hz).

(b) Second mode shape, $\mathrm{f}=1650.80 \mathrm{~Hz}$.

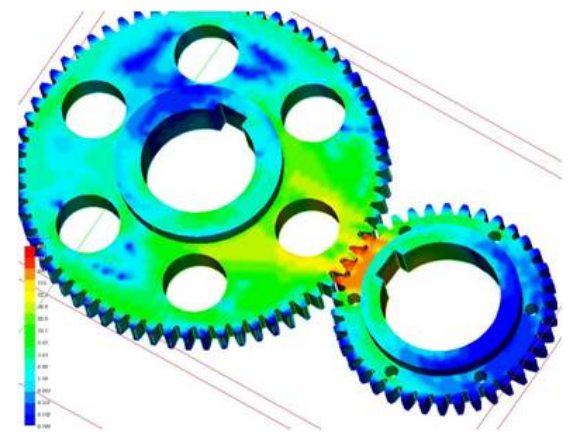

Figure 5: Strain distribution for $\mathrm{t}=0,02$

We have further analyzed strain conditions demonstrated in the gear set, and the results are shown in Fig. 5.

Using the above procedures, the results are obtained for the geared transmission on the Fig. 2a, using transmission parameters given in Table 2.

Table 2: Data for gear transmission on the figure 3

\begin{tabular}{|r|r|r|r|r|}
\hline Parameter & Gear 1 & Gear 2 & Gear 3 & Gear 4 \\
\hline Number of teeth & 27 & 30 & 33 & 22 \\
\hline Modul (m) & 0.003 & 0.003 & 0.003 & 0.003 \\
\hline Pitch circle diameter (m) & 0.081 & 0.090 & 0.099 & 0.066 \\
\hline Addendum circle diameter (m) & 0.087 & 0.096 & 0.106 & 0.072 \\
\hline Base circle diameter (m) & 0.073 & 0.0828 & 0.0918 & 0.0588 \\
\hline Measurement teeth number (m) & 0.004 & 0.004 & 0.004 & 0.003 \\
\hline Standard angle (degrees) & 20 & 20 & 20 & 20 \\
\hline Axial distance (m) & 0.081 & 0.081 & 0.081 & 0.099 \\
\hline
\end{tabular}


Transmission load distribution along the line of contact for each transmission gear pairs given on Fig. 6, and distribution of loads on the transmission elements for $t=0.02$ is given on the Fig. 7 as an output from the nonlinear dynamic analysis of the geared transmission.

Additional results for strain distribution along the line of contact for each gear par is given on the Fig. 8.

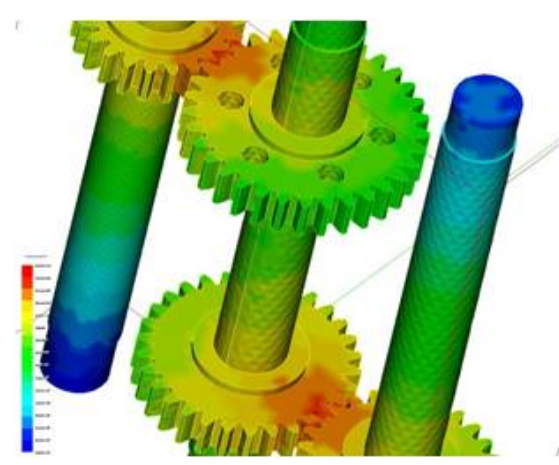

Figure 6: Transmission load distribution in time step $\mathrm{t}=0.02$

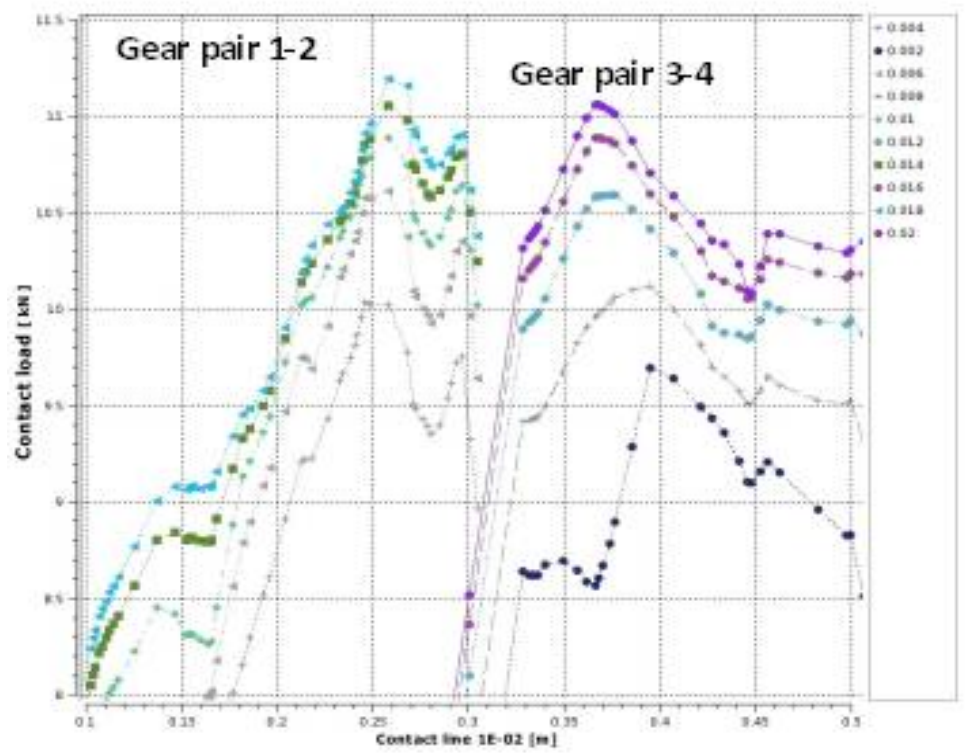

Figure 7: Load distribution along the line of contact for each transmission gear par $(t=0 \div t=0.02)$ 


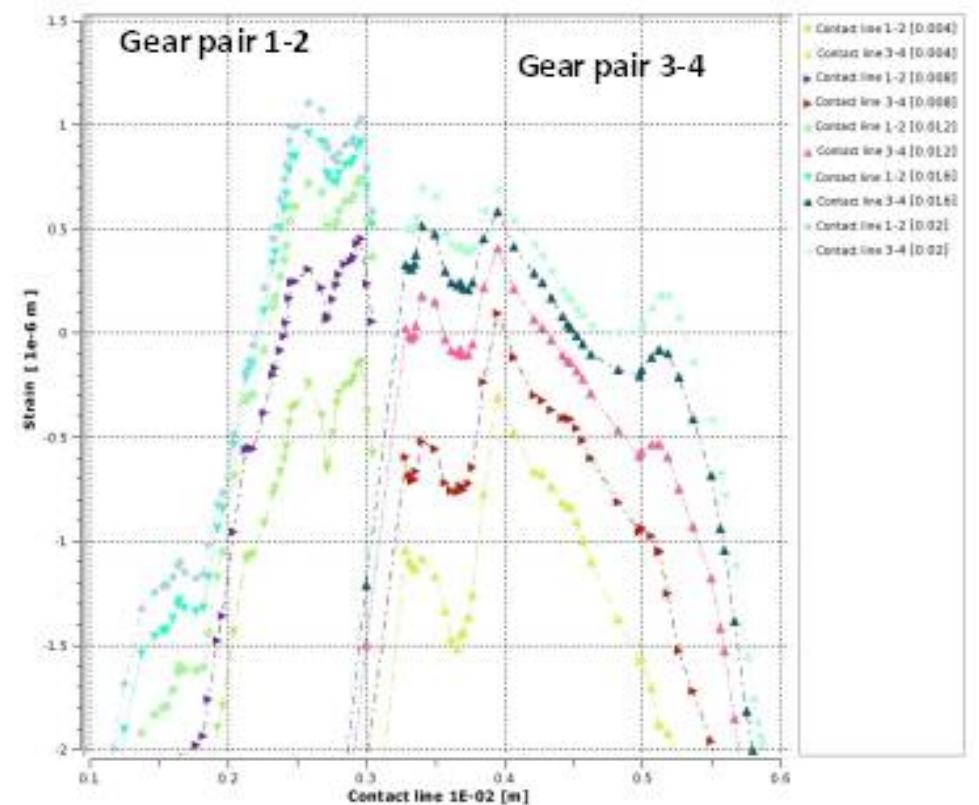

Figure 8: Strain distribution along the line of contact for each transmission gear par $(t=0 \div t=0.02)$

\section{Conclusion and guidelines for further research}

In this paper the basic principles of modal analysis are given. Using basic laws of mathematics and mechanics, it is possible to describe the dynamic behavior of a system. The solution of differential equations, can be obtained using numerical methods.

So, Finite Elements Method is used in this paper to develop the real model of the geared set. On the basis of the results shown in this paper it has been concluded that the methodology developed to study the dynamic behavior of complex systems is very efficient. It gives a lot of possibilities and can be easily upgraded for analysis of other effects.

The modal analysis and analysis of the stress and strain conditions that suggest that the system of only two gears is very complex and that it is almost impossible to include all the effects by such and similar research.

RESULTS were obtained using the computer program COSSMOS M.

Further research should be directed at studying the effects of mutual dynamic impact of teeth in engagement, as well as at including connection between the shaft and gear into the dynamic model and the like. 


\section{References}

[1] Zienkiewics O.C, (1977) The finite Element Method, Third edition, mcGraw-Hill, London.

[2] Kahraman, A., Singh, R. (1990): Non-linear Dynamics of a Spur Gear Pair, Journal of Sound and Vibration, 142(1), 49-75.

[3] Parker, G.R., Agashe, V., Vijayakar, M.S. (2000): Dynamic Response of a Planetary Gear System using a Finite Element/Contact Mechanics Model, Transactions of the ASME, 122, 304-310.

[4] Nikolic, V. (1999). Mechanical analysis of gears. Faculty of Mechanical Engineering, Cimpes, Kragujevac .

[5] Diana Ć. Dolićanin-Đekić, Igor A. Soloviev, Radosav Ž. Đorđević, Gradimir V. Milovanović, Sistemi diferencijalnih jednačina, D , 2013.

[6] Dimitrijević D., Nikolić, V., (2008) Eigenfreuqency analysis of the spur gear pair with moving excentric masses on the body of one of the gears, FME Transaction, Beograd.

[7] Atanacković, M.T., Dolićanin, Ć.D., Pilipović, S. (2011). Forced Oscillations of a Single Degree of Freedom System with Fractional Dissipation. Scientific Publications of the State University of Novi Pazar, Series A: Applied Mathematics, Informatics and Mechanics, vol. 3, no. 1, p. 1-11.

[8] Hedrih (Stevanovic), K.; Nikolic-Stanojevic, V. (2010) A Model of Gear transmission fractional order System dynamics, (Research Article) // Mathematical Problems in Engineering, Received 24 January (2010); Revised 2 March; Accepted 6 May (2010), (http://www.hindawi.com/journals/mpe/aip 972873.html), ISSN 1024-123x.

[9] Nikolić, V., Dolićanin, Ć., Dimitrijević, D. (2010). Numerical modelling of gear set dynamic behaviour. Scientific Technical Review, no. 3-4, p. 48-54.

[10] Nikolić, V., Dolićanin, Ć., Dimitrijević, D. (2012). Dynamic Model for the Stress and Strain State Analysis of a Spur Gear Transmission, Strojniki vestnik - Journal of Mechanical Engineering, Vol 58, No 1, 2012, pp 56-67.

[11] Parker, G.R., Vijayakar, M.S., Imajo, T. (2000). Non-linear dynamic response of a spur gear pair: modelling and experimental comparisons. Journal of Sound and vibration, vol. 237, no. 3, p. 435-455. 